\title{
Estimation of heart rate from foot worn photoplethysmography sensors during fast bike exercise
}

\author{
Delaram Jarchi and Alexander J. Casson, Senior Member, IEEE
}

\begin{abstract}
This paper presents a new method for estimating the average heart rate from a foot/ankle worn photoplethysmography (PPG) sensor during fast bike activity. Placing the PPG sensor on the lower half of the body allows more energy to be collected from energy harvesting in order to give a power autonomous sensor node, but comes at the cost of introducing significant motion interference into the PPG trace. We present a normalised least mean square adaptive filter and short-time Fourier transform based algorithm for estimating heart rate in the presence of this motion contamination. Results from 8 subjects show the new algorithm has an average error of 9 beats-per-minute when compared to an ECG gold standard.
\end{abstract}

\section{INTRODUCTION}

Recently highly portable non-obtrusive wearable heart rate monitors have become readily available, and are now used in a number of applications. These range from non-healthcare situations, such as the heart rate monitoring of athletes during training, to at-home rehabilitation, for example monitoring the heart rate of rehabilitation subjects as they perform different exercises [1]. In many of these emerging wearable heart monitoring applications the devices are used out-ofthe-clinic, in uncontrolled environments, and the collected signals are heavily contaminated by motion artifacts.

For measuring heart rate, electrocardiogram (ECG) signals from the chest have the maximum signal strength and robust signal processing algorithms can be applied for the reliable estimation of heart rate parameters during physical exercise. However, as a recording modality it has a number of limitations: ECG electrodes are relatively difficult for a non-specialist to attach correctly and in the right place; the presence of chest hair decreases the quality and longevity of the electrode connection; and the chest is a relatively intimate place in which to site a sensor. Many users prefer non-invasive heart measurements to be performed from more peripheral sites such as the wrist.

Peripheral wearable heart monitoring is possible by using photoplethysmography (PPG) to measure changes in blood flow by shining a light source into the body [2]. The method can be used in many parts of the body, such as the forehead [3] and ear [4], and is very popular in emerging smartwatch devices placed on the wrist. However, the PPG recording modality has two significant limitations: PPG signals are highly corrupted by motion artifacts [5] which historically have restricted its use to no-motion situations; and the

This work was supported by the Engineering and Physical Sciences Research Council grant number EP/M009262/1.

D. Jarchi and A. J. Casson are with School of Electrical and Electronic Engineering, University of Manchester, Manchester, UK. \{delaram.jarchi, alex.casson\}@manchester.ac.uk light source requires a large amount of power (typically approximately $1 \mathrm{~mW}$ ) limiting the operational life of highly miniaturized wearables with small batteries.

To overcome the motion artifact challenge recently several signal processing algorithms have been reported for extracting the average heart rate from a motion contaminated PPG signal collected under exercise. For example, proposed methods are based on Empirical Mode Decomposition (EMD) [6], Independent Component Analysis (ICA) [7], Kalman filtering [8], adaptive filters [3], [9] and sparse signal reconstruction [10]. These have shown to be to able to extract the average heart rate from a motion contained PPG trace to within (approximately) \pm 5 beats-per-minute (bpm).

In this paper we use these advances in signal processing to tackle the second challenge, that of power consumption. Energy harvesting, where the batteries present are supplemented by collecting the intrinsic energy available in the environment, is critical for use in future wearable devices as it is the only method for creating sensors which are truly power autonomous and can go beyond the limited lifetimes provided by batteries. However extracting large levels of power from miniature harvesters, sufficient to power a wearable sensor, is very difficult.

Our new approach aims to overcome this by recognizing that the largest amount of energy, by far, is available by placing the harvester device on the lower half of the body [11]. We thus propose to locate the wearable PPG sensor on the foot/ankle periphery, rather than the wrist periphery, where it can make use of this energy harvesting potential and enable truly long-term and autonomous operation despite the high power light source required. Doing this successfully first requires removing motion interference from the foot/ankle PPG trace. This is a substantially more challenging case than the wrist PPG examples cited above due to the larger and more impactful movements by the legs during exercise.

This paper presents a new artifact removal algorithm based upon a normalised least mean square adaptive filter and tracking method for estimating average heart rate from a foot/ankle located PPG sensor while under motion. Subjects were equipped with wrist and ankle worn sensors and performed physical exercises such as walking/running on a treadmill and cycling using an exercise bike to evaluate the PPG signals, motion corruption, and performance of the removal algorithm under situations where motion can heavily influence the PPG signals collected. Section II describes the experimental setup used and the algorithm proposed for the estimation of average heart rate. Section III analyses and discusses the performance of the algorithm. 


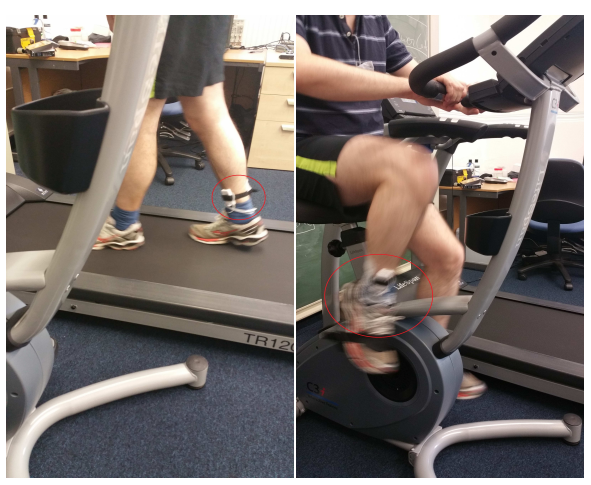

(a)

(b)

Fig. 1. Subject wearing the PPG sensor during: (a) walking, (b) cycling.

\section{METHODS}

\section{A. Experimental setup}

Eight healthy subjects aged 24-32 participated in an experiment to collect ankle/foot PPG signals under motion, having a groundtruth ECG signal collected from the chest. All procedures in this study were reviewed and approved by the University of Manchester Research Ethics Committee.

PPG and simultaneous three-axis accelerometer signals were collected using Shimmer 3 [12] devices. In Fig. 1, one subject wearing foot and wrist sensors during walking on the treadmill and cycling on the bike is shown. A simultaneous ECG trace was also collected using a Cam $n$ Tech platform [13]. Participants were asked to use a treadmill and exercise bike at two self-selected speed of low and high. Each session of slow/fast walking/biking lasted for a maximum of ten minutes. After each session the subjects remained seated and relaxed for 10 minutes in order for the heart rate to come back to a normal rate for the rest state. The wrist/foot worn PPG and accelerometer signals were recorded simultaneously at $256 \mathrm{~Hz}$ and manually synchronized with the ECG signals.

\section{B. Estimation of average heart rate}

The heart rate extraction method in this paper is similar to the recent proposed method in [9] for wrist PPG signals, modified to remove the motion artifacts obtained at the foot/ankle. Fig. 2 gives an overview of our method.

The raw PPG and co-located accelerometry signals are first band-pass filtered (3rd order Butterworth, 0.5-20 Hz) and down-sampled by a factor $D$ (default $D=10$ ), and passed to three Normalised Least Mean Square (NLMS) adaptive filters, one for each axis of acceleration data. The NLMS adaptive filters are implemented such that the desired signal is the PPG trace and the filter input is the accelerometer trace. The objective is to adaptively estimate a motion-free PPG signal by suppressing the signal components measured by the accelerometers. To do this the measured PPG signal $p(n)$ is modeled by:

$$
p(n)=\widetilde{p}(n)+m(n)+v(n)
$$

where $\widetilde{p}(n)$ is the motion interference free PPG signal, $m(n)$ is the motion artifact and $v(n)$ is the residual sensor

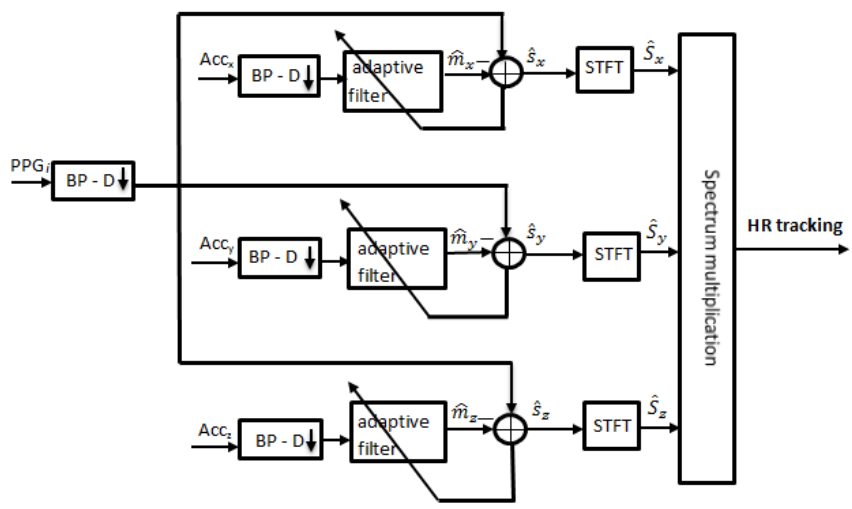

Fig. 2. Overview of artifact removal NLMS algorithm based on adaptive filters.

recording noise. The NLMS filter models the motion artifact as a linear function of the accelerometer data

$$
m(n)=\mathbf{h}^{T}(n) \mathbf{a}(n)
$$

where $\mathbf{h}$ is an unknown transfer function. Therefore an error signal is defined as:

$$
e(n)=p(n)-\mathbf{h}^{T}(n) \mathbf{a}(n) .
$$

and a linear update equation can be used to iteratively estimate $\mathbf{h}$ from samples of $p(n)$ and $\mathbf{a}(n)$ by minimizing $e(n)$. This update procedure is formulated as:

$$
\mathbf{h}(n+1)=\mathbf{h}(n)+\frac{\mu(n)}{\|\mathbf{a}(n)\|^{2}} \mathbf{a}(n) e(n)
$$

where $\mu(n)$ is an update step size parameter. Here $\mathbf{a}(n)$ is a vector of length $L$ (filter order) of acceleration samples. Knowing $\mathbf{h}$ an estimate of the motion interference in the PPG trace, $\hat{m}_{x, y, z}$, can be generated and is subtracted from the recorded PPG trace $p(n)$. This procedure is applied separately for the three acceleration axes, producing three estimates of a motion-reduced PPG trace, $\hat{s}_{x, y, z}$.

Estimation of heart rate is then performed in the frequency domain using the Short Time Fourier Transform (STFT) to first extract the frequency components in each signal $\hat{s}_{x, y, z}$. To keep the dominant frequency components from each motion-reduced PPG signal a single combined spectral signal is obtained by multiplying the three individual spectrums, and then taking a cube root:

$$
\widetilde{S}(n, f)=\sqrt[3]{\Pi_{i=1}^{3} S_{i}(n, f)} .
$$

$S_{i}(n, f)$ is the estimated spectrum of the motion-reduced PPG signal using a combination of each accelerometer axis and the measured PPG signal, and $\widetilde{S}(n, f)$ is the final estimated spectrum. Here the NLMS filter order is set to 9 , the step-size of $\mu$ has been set to 0.1 and the number of FFT points for the STFT algorithm has been chosen as 4096 .

The end estimate of the heart rate in beats-per-minute (bpm) is taken as the highest power frequency in $\widetilde{S}(n, f)$, with this frequency converted from $\mathrm{Hz}$ to bpm. To avoid spurious peaks and infeasible step changes in heart rate estimates between analysis windows an HR tracking algorithm has been implemented which follows the frequency trace 

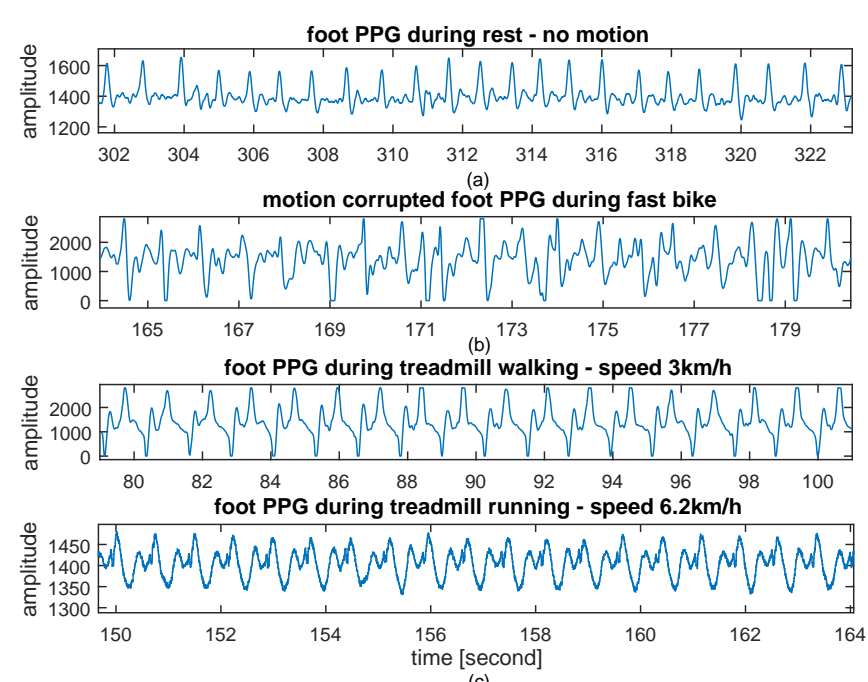

(c)

Fig. 3. Example PPG signals from the ankle/foot: (a) at rest, (b) while biking, (c) while walking/running.

with maximum energy along the spectrum in each analysis window. This limits the reported heart rate to a range of $60 \mathrm{bpm}$ to $140 \mathrm{bpm}$ with no jumps greater than $14 \mathrm{bpm}$ between consecutive time windows. In our current set up an overlapping window of 6 second duration, updating every 2 seconds in 2 second steps, has been used.

\section{Comparison method}

The foot/ankle PPG heart rate values are compared to a gold standard measurement from the chest ECG trace. By estimating and locating the R-peaks in the ECG signals it is possible to estimate the number of beats per minute. To have a pairwise comparison we used the same method as in Fig. 2, without the adaptive filter part, to extract the heart rate from the ECG trace. The raw ECG data is down-sampled $(D=5)$ and the STFT and heart rate tracking algorithms then operate as described above.

\section{RESULTS AND DISCUSSION}

\section{A. Signals during rest}

In Fig. 3, examples of foot/ankle PPG data are shown to illustrate the typical signal morphologies in the no-motion case and the typical motion artifacts that are encountered. Fig. 3(a) shows a case of PPG signals collected when no motion is present. A very clear signal is seen, with clear peaks due to each heart beat. This makes it possible to extract the heart rate without further sophisticated signal processing. Such a case is shown in Fig. 4 which illustrates data for one subject while in the rest period after walking on the treadmill. The spectrum of the ECG signal is provided in Fig. 4(a), with a segment of the raw time domain signal in Fig. 4(c). These can be compared with Fig. 4(b) and (d) which show the equivalent traces for the foot/ankle PPG recording. Note that these figures are for the raw collected signal. During the rest period there is little/no motion artifact affecting the signals and the algorithm from Section II has not been applied. In Fig. 4(e), the average heart rate in terms of bpm has been shown, and demonstrates a good estimation of heart rate from
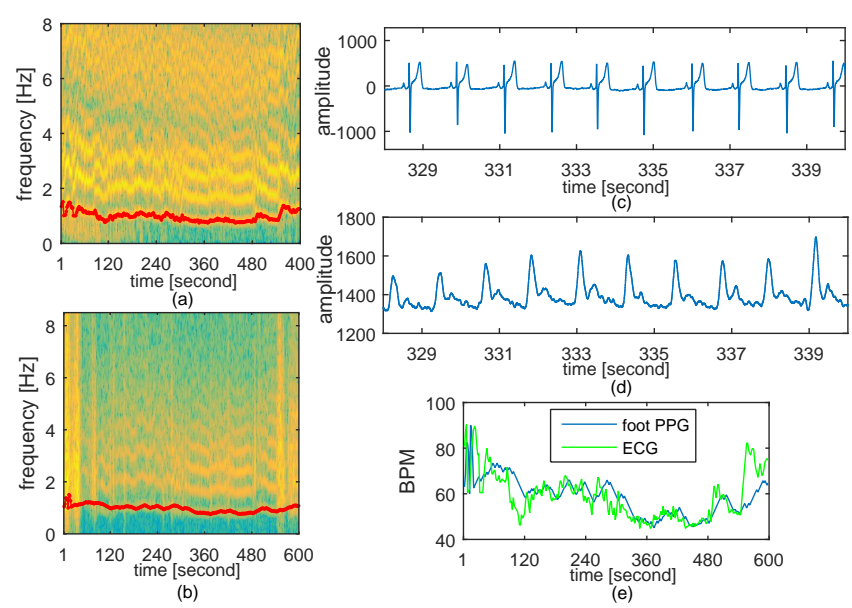

Fig. 4. (a) Spectrum of ECG signal at rest. The estimated heart rate is shown in red color. (b) Spectrum of the PPG signal at rest. It can be seen the spectrum is noisy for the beginning and end of the data. This is due to slight motion and transition of the subject from an activity to rest and vice versa. (c) A segmented ECG signal shows clear motion free and regular heart beats. (d) The foot worn PPG signals. (e) Estimated BPM from ECG signals and PPG signals for 10 minutes data at rest session.

PPG and ECG signals in the middle of rest period. A poor agreement between the PPG and ECG estimated heart rates is seen at the start and end of Fig. 4(e). This is due to the presence of motion artifacts as subject goes from walking on the treadmill into the seating position at the beginning of rest period and preparing for a new session at the end of rest period, and these artifacts have not been corrected for.

\section{B. Signals during biking}

Fig. 3(b) shows PPG signals affected by motion artifact during fast bike riding exercise. Significant time domain corruption of the signals is readily apparent, and it is not feasible to extract the heart rate from the foot/ankle PPG trace in the time domain, necessitating the new artifact removal approach introduced in Section II.

The performance of this with one subject is illustrated in Fig. 5 for the fast bike riding case. Fig. 5(a) shows the frequency spectrum of the raw PPG, which contains a large number of high frequency components which are not of physiological origin. These are suppressed, although not removed entirely, in Fig. 5(b) once the adaptive filtering algorithm has been applied, and the heart rate can then be tracked as shown in Fig. 5(d).

Fig. 5(g) shows the end comparison of heart rates estimated from chest ECG and foot/ankle PPG for this example. After approximately two minutes a good agreement between the two measures is obtained. (This agreement will be quantified in Section III-D.) The two minutes corresponds to the convergence/run-in time of the adaptive filter, which requires a number of PPG and accelerometer samples in order to build an accurate model of the current $\mathbf{h}$ transfer function. This latency in producing an accurate heart rate estimate is an inherent limitation of our current approach.

\section{Signals during walking/running}

Fig. 3(c) shows the signals from one subject during walking and running on a treadmill, with time domain corruption introduced due to the presence of motion. Nevertheless Fig. 6 

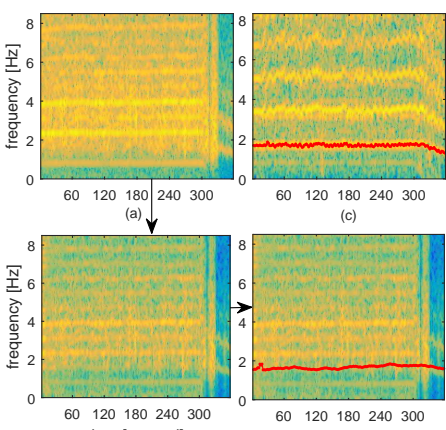

time [second]

(d)

(b)

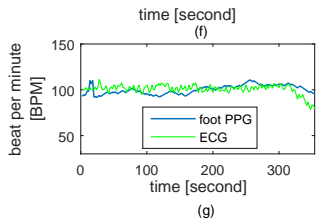

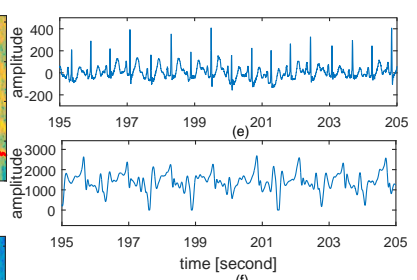

Fig. 5. (a) Spectrum of PPG signal corrupted by the motion artefact. (b) Reestimation of the PPG spectrum after applying adaptive filter and motion artefact reduction where estimated heart rate is shown in red color in (d). (c) Spectrum of ECG signal and the estimated heart rate in red color. A segmented ECG signal (e) and foot PPG signal (f) during walking on the treadmill. (g) The estimated BPM from ECG signals and PPG signals for about 5 minutes data during walking on the treadmill.
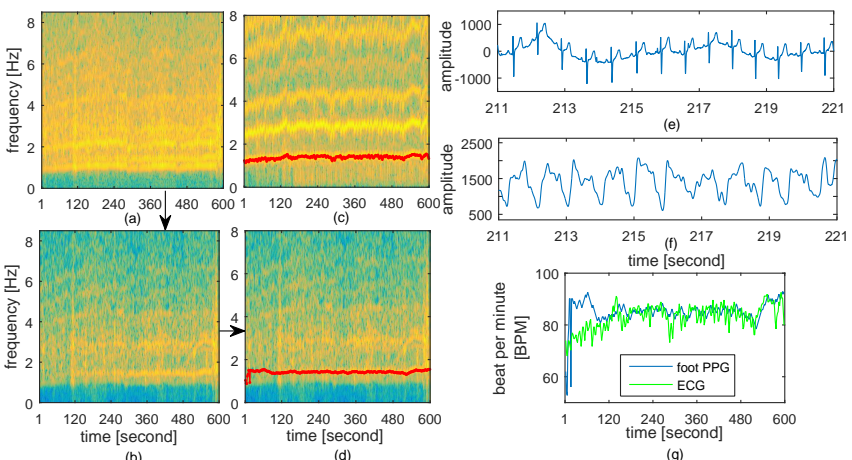

Fig. 6. (a) Spectrum of PPG signal corrupted by the motion artefact. (b) Reestimation of the PPG spectrum after applying adaptive filters and motion artefact reduction estimated heart rate in red color in (d). (c) Spectrum of ECG signal and the estimated heart rate is in red color. A segmented ECG signal (e) and foot PPG signal (f) during fast bike activity. (g) The estimated BPM from ECG signals and PPG signals for 10 minutes data during fast bike exercise.

presents results for the algorithmic cleaning of the PPG trace in order to produce a heart rate estimate which is comparable to that from ECG, Fig. $6(\mathrm{~g})$. The agreement in this case is worse than that for the biking case in Fig. 5. It is apparent that customized adaptive filters will be required for each activity, and further work on the filter for the walking/running case is required to obtain a satisfactory agreement between the PPG and ECG cases.

\section{Quantification of performance}

For the 8 subjects studied, the PPG signals during fast bike exercise are used to estimate the average heart rate, and these are compared to the average heart rate from the ECG signals, with the results given in Fig. 7. This shows the rootmean-squared-errors between the beats-per-minute calculated using the ECG and PPG input traces. The mean and standard deviation of the root mean squared error has been obtained as $9.1 \pm 3.1 \mathrm{bpm}$, with the algorithm performing much better in some subjects than others. The average of approximately $9 \mathrm{bpm}$ is satisfactory as an initial algorithm development to prove the concept of foot/ankle PPG, and we are confident the error it can be reduced by improving the HR tracking algorithm and adaptive setting of system parameters before and after applying adaptive filters in future studies.

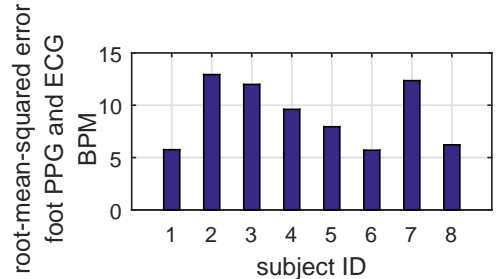

Fig. 7. Root-mean-squared-error of average heart rate in terms of bpm between estimations from the ECG and foot PPG sensors during fast bike activity.

\section{CONCLUSIONS}

This paper has proposed a NLMS adaptive filter and STFT based algorithm for estimating the average heart rate from a PPG sensor placed on the foot/ankle during physical exercise. Placing the sensor in this location could help power the device by using energy harvesting, but it results in significantly more motion artifacts in the collected PPG trace. Our algorithm, assessed using data from 8 participants in a fast biking situation, shows an average heart rate estimation error of $9 \mathrm{bpm}$. At present this performance is limited by particularly heavy motion artifacts which are seen in the PPG signals of a few subjects, and by the convergence/run-in time of the adaptive filter which increases the error when the algorithm is first turned on. We expect to improve the algorithm proposed in this study by modeling the motion artifact from the acceleration signals using nonlinear techniques and also to use an adaptive method to update the parameters of the system for each subject separately.

\section{REFERENCES}

[1] J. Park, J. Cho, T. Nam, and J. Choi, "A unconstrained multi-channel heart rate monitoring system for exercising rehabilitation patients," IEEE EMBC, pp. 3512-3515, 2007.

[2] J. Allen, "Photoplethysmography and its application in clinical physiological measurement," Physiol. Meas, vol. 28, no. 3, pp. R1R39, 2007.

[3] S. H. Kim, D. W. Ryoo, and C. Bae, "Adaptive noise cancellation using accelerometers for the PPG signal from forehead," IEEE EMBC, 2007.

[4] E. S. Winokur, D. D. He, and C. G. Sodini, "A wearable vital signs monitor at the ear for continuous heart rate and pulse transit time measurements," IEEE EMBC, pp. 2724-2727, 2012.

[5] R. Krishnan, B. Natarajan, and S. Warren, "Two-stage approach for detection and reduction of motion artifacts in photoplethysmographic data," IEEE Trans. Biomed. Eng., vol. 8, no. 57, pp. 1867-1876, 2010.

[6] X. Sun, P. Yang, Y. Li, Z. Gao, and Y.-T. Zhang, "Robust heart beat detection from photoplethysmography interlaced with motion artifacts based on empirical mode decomposition," IEEE-EMBS BHI, 2012.

[7] B. S. Kim and S. K. Yoo, "Motion artifact reduction in photoplethysmography using independent component analysis," IEEE Trans. on Biomed. Eng., vol. 53, no. 3, pp. 566-568, 2006.

[8] B. Lee, J. Han, H. J. Baek, J. H. Shin, K. S. Park, and W. J. Yi, "Improved elimination of motion artifacts from a photoplethysmographic signal using a kalman smoother with simultaneous accelerometry," Physiol. Meas, vol. 31, no. 12, pp. 1585-1603, 2010.

[9] T. Schack, C. Sledz, M. Muma, and A. M. Zoubir, "A new method for heart rate monitoring during physical exercise using photoplethysmographic signals," EUSIPCO, pp. 2716-2720, 2015.

[10] Z. Zhang, Z. Pi, and B. Liu, "TROIKA: A general framework for heart rate monitoring using wrist-type photoplethysmographic signals during intensive physical exercise," IEEE Trans. on Biomed. Eng., vol. 62, no. 2, pp. 522-531, 2015.

[11] P. D. Mitcheson, E. M. Yeatman, G. K. Rao, A. S. Holmes, and T. C. Green, "Energy harvesting from human and machine motion for wireless electronic devices," Proc. IEEE, 2008.

[12] "http://www.shimmersensing.com," 2015.

[13] "http://www.camntech.com/," 